\title{
Domain knowledge for surveillance applications
}

\author{
Lauro Snidaro, Massimo Belluz, Gian Luca Foresti \\ Department of Mathematics and Computer Science \\ University of Udine, Italy \\ Email: \{snidaro\}@dimi.uniud.it
}

\begin{abstract}
In this paper, we address the problem of representing domain knowledge for situation awareness in a security application. While ontologies are appropriate for describing taxonomical knowledge, they cannot express more complex knowledge such as entailments. In this paper, we describe how domain knowledge can be encoded through OWL ontologies and SWRL rules in order to reason about the entities and their interactions in a surveillance application. We describe how events can be described through ontologies and how video sequences can be annotated using the MPEG-7 standard.
\end{abstract}

Keywords: Ontologies, situation awareness, information fusion, video surveillance.

\section{INTRODUCTION}

Recent research on automatic surveillance system is focusing on the development of languages, methodologies, and tools to represent and manage domain knowledge [1] [2]. This effort is geared to instill some form of intelligence into nowadays systems, in order to automatically recognize the entities, their actions and interactions, in the observed environment [3]. In other words, the goal is to have systems that should be able to continuously assess the state-of-things in the monitored areas, and recognize possible threats or dangerous conditions.

Generally, these systems have to operate in crowded and complex environments where anomalous events have to be distinguished from the normal course of actions. One way to infer the behaviour of the observed objects is by analysing their movements [3]. Several techniques have been proposed in the past, but all of them can be categorized into two main classes. The first one addresses the problem of modelling behaviour by statistically analysing trajectories [4]. By this approach, the system automatically learns the common patterns of activity in the observed environment. Anomalies are than detected as something that differs from the learnt model. The main advantage of this class of solutions is that the system is dynamic, since the learning phase is mostly automatic, thus easing its deployment. The main disadvantage is that this approach cannot cater for a priori knowledge that one may want to convey to the system.

The second category is comprised by solutions that model anomalous behaviours explicitly. In [5], for example, events are modelled and recognized through the use of context-free grammars. Systems that rely on this approach can be deployed much less easily, as they require a set-up phase where an operator has to define the events of interest.

Ontologies provide a principled way to encode domain knowledge into current applications [6]. They can be used to describe the entities and the relations between them. Through ontologies, the a priori knowledge can be formally detached from the application code, thus facilitating the design and development of intelligent systems. In addition, since the standardization of the Web Ontology Language (OWL) by the $\mathrm{W} 3 \mathrm{C}$, they have constantly gained interest and their usage has become widespread. However, OWL can be useful for describing taxonomical knowledge, but it shows all its limitations when other forms of knowledge have to be included in the system. For example, it cannot encode rules to derive new knowledge if one or more conditions occur. This is particularly limiting in a surveillance scenario, where one would like to encode a complex event (i.e. identity fraud) as the temporal sequence of a series of simple events (i.e. person approaches door, badge is swiped, etc.).

A solution can be found by encoding rule-based knowledge with the Semantic Web Rule Language (SWRL). Even though it is still in development, together with OWL, it may provide a possible solution to encode the domain knowledge needed to assess the current situation, as described in [7]. SWRL, infact, is natively integrated into the Protégé editor and this facilitates the duty of the operator that has to encode the rules.

In this paper, we describe how OWL+SWRL can be applied in the context of surveillance applications. In particular, we show how a complex event can be described by composing a sequence of simple events, thus fusing together different information. In addition, we show how video streams can be annotated, using the MPEG-7 sintax, with the instances of detected entities and events. MPEG-7 is a multimedia content description standard. It defines the syntax to encode information about the content of a document (metadata), not the content itself (data). Here, we employ the standard to generate XML files describing events and their semantics according to ontologically defined domain knowledge. These annotations can be transmitted over the network in order to exchange information between the processing units of a large and distributed surveillance system.

\section{EVENT REPRESENTATION}

Building a situational picture requires a system to be able to assess the current state of the observed environment. More specifically for security purposes, the system should be able to detect and recognize events. These can be subdivided in simple and complex. The former ones can be considered as the variation of an entity's state, while the latter are a sequence of atomic activities. In the surveillance domain, 
several attempts have been made to model events: in [8] Coupled Hidden Markov models (CHMM) are used to learn interaction semantics, in [5] Hidden Markov Models are coupled with context-free grammars, in [2] hierarchical decomposition and single/multiple thread terminology, to describe single and concurrent events, are advocated. The Video Event Representation Language (VERL) to explicitly manage the semantics of events and to instruct the system to recognize them, is presented in [1].

This language, represents a convenient way to express the knowledge of the system on relevant activities in the observed environment. Indeed, the system can be explicitly taught to discriminate between normal and anomalous behaviour. Listing 1 shows how an "Identity fraud" event can be defined.

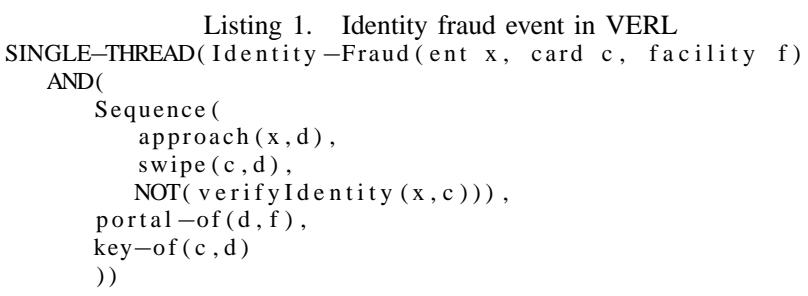

In the example above, the malevolent act of attempting to gain entry into a protected facility through a stolen access card is described. The event takes place when entity $x$ approaches door $d$ of facility $f$, card $c$ is swiped at $d$ (and $c$ is a valid card to get into $f$ through $d$ ), but the biometric control subsystem fails to verify the identity contained in $c$ with the biometric features extracted from entity $x$.

The VERL language constitutes a potentially interesting choice for event representation, however there is no working implementation of it. The level of expressiveness of VERL requires infact the OWL-Full declination of the OWL language which is not computable. There exists a partial implementation that defines some of the syntactic elements of VERL in OWL, however, there is no mechanism that defines complex events and no mention on how they should be derived from instances of simple events.

\section{A. Rule languages}

OWL is an appropriate language for expressing taxonomies in terms of classes, subclasses, and relations between them. However, it falls short in describing rules that can be computed by a reasoner to derive knowledge. Rule-based systems are successfully employed across a variety of domains (e.g. economic models, clinical diagnoses, process control) in a multitude of implementations with very limited support for interoperability. The widespread interest on ontologies and means to exchange knowledge bases has recently pushed the endeavours toward the adoption of a common rule base that could be computed by different rule engines. The effort has led to the development of several rule languages such as RuleML, SWRL, Metalog, and ISO Prolog among others [9].

The Semantic Web Rule Language (SWRL) is intended to be the rule language of the Semantic Web. It is based on OWL DL, OWL Lite, and the Rule Markup Language. All rules are expressed in terms of OWL concepts (classes, properties, inviduals), and this allows the integration of taxonomic knowledge and rule bases about a given domain under the common hood of OWL. This means that rules can be used to infer new knowledge from existing OWL ontologies. The widespread adoption of OWL is confirmed by the availability of a multitude of editors to help build OWL ontologies. In particular, the already mentioned Protégé editor provides a "SWRLTab" to assist the definition of SWRL rules [9].

The following logic expression is an example of a SWRL translation for the complex event described in Listing 1:

$$
\begin{array}{r}
\text { Person }(? x) \wedge \operatorname{Door}(? y) \wedge \text { isCloseTo }(? x, ? y) \wedge \\
\wedge \operatorname{Card}(? z) \wedge i s \text { SwipedOn }(? z, ? y) \wedge i \text { isOpenedBy }(? y, ? z) \wedge \\
\wedge i s N o t \text { VerifiedBy }(? x, ? z) \Rightarrow \\
\Rightarrow \text { isViolating }(? x, ? y)
\end{array}
$$

Since this expression is eventually translated into OWL by the SWRLTab editor, properties of involved entities can be seamlessly retrieved through formal or domain ontologies. The above rule constitutes a hypothetical proposition where the antecedent is given by the conjunction of several predicates. If all the predicates in the antecedent are verified, that is, the video-surveillance system reports that a person $x$ is close to a door $y$, and a magnetic card $z$ is swiped on door $y$, and $z$ is a valid card for door $y$, but the identity stored on the card is not matching the one provided by the biometric control system failing to recognize $x$, then the consequent follows verifying the predicate that the person $x$ is attempting an unauthorized access to door $y$.

The SWRLTab Protégé plug-in also provides high level Java APIs called "SWRL Factory" that allow the creation and modification of SWRL rules programmatically. In this way, external applications can access/create/modify/delete rules in the rule base. It should be noted that the APIs don't provide inferencing capabilities. Inferencing is provided by the external reasoner Jess $^{1}$ through the "SWRL Bridge" which is part of the ProtégéOWL APIs. These APIs allow the interaction with the rule engine and can be used to compute inferences.

Note that rules such as (1), which exemplifies the definition of the complex event "Identity fraud" for a surveillance system in an Intelligent Building, provide an effective way of integrating knowledge coming from heterogeneous sources. In the example, the antecedent is composed by atomic (or simple) events detected by different types of sensors (e.g. surveillance camera, magnetic card reader, biometric system). The recognition of atomic events can be performed per sensor through techniques such as bayesian networks [2]. A number of features can be extracted from the data produced by a sensor (e.g. the position of a person can be extracted from the video stream provided by a surveillance camera) and they can be used to build bayesian networks for each atomic event of interest. Each network will provide a boolean output stating if the atomic event has occurred or not. These binary outputs

\footnotetext{
${ }^{1}$ http://www.jessrules.com/
} 
will then be fed to the reasoner to check if any rule in the rule base can be fired.

\section{B. Uncertainty}

As discussed in the previous Section, complex events are defined through logic expression that can assume only boolean values. Indeed, the verification of atomic events is expressed by predicates and this could be a limiting condition in many real world scenarios. Whatever technique is used to recognize atomic events, then its outputs have to be, in most of the cases, thresholded to yield a boolean value. Take for example the proximity condition expressed by the predicate isCloseTo in (1). To detect the atomic event that a person is close to a given object, the current distance between the person and the object must be compared against a preset threshold. In real world scenarios, hard thresholds are troublesome in the vast majority of the cases. In the example above, rule (1) would not fire if the video-surveillance system had over-estimated the distance between the person and the door yielding a value above the threshold.

It would be better then to assign a degree of truth to expressions such as the antecedent of (1), instead of computing its boolean value. This can be done by assigning probability coefficients to each term, and than evaluating the overall degree of truth of the weighted expression. A fuzzy approach can also be foreseen by exploiting a specific extension of the Jess engine called "FuzzyJess" [10], that provides the functionalities needed to handle fuzzy logic.

\section{EVENT ONTOLOGY}

A key point in the proposed approach is how an event should be described within an ontology to ease the formalization of contextual knowledge in a surveillance scenario. On one hand, the definition should be expressive enough to express all the knowledge that can be exploited for security purposes in the given scenario. On the other hand, the definition should not be overly complex in order to be readable, usable, and maintainable by a human operator.

The typical class/subclass specialization mechanism allows the definition of a hierarchy of events. The following are some of the properties, common to the "Event" superclass, that we have defined in our experiments:

- UID [datatype int]: Unique numerical identifier assigned to every event instance. It is used both by inferential rules and by the application that has to manage and populate the properties of all the instances of the class relating to detected events.

- Activation [datatype datetime]: Timestamp of the activation time. It indicates the time instant at which the event has been detected. This is a mandatory property as it used to reason about the relations occurring in a temporal sequence of events.

- Duration [datatype float]: Event duration in seconds. The value 0 is used for instantaneous events and when the property doesn't apply. Used for a posteriori reasoning about a chain of events.
- Subject [object reference]: Refers to the instance of the entity that is the "subject" of the event. Depending on the type of event, it may refer to the agent that initiates the event, or to the entity that causes the event. To better capture the possible semantics, the property is specialized into:

- Agent [object reference] The agent is animate and initiates the action intentionally.

- Causer [object reference] The causer generates the event unintentionally.

It should be noted that the two disjoint specializations of the "Subject" class are not exhaustive. It is therefore legal to use "Subject" when the two specializations don't apply.

From the "Event" superclass, three subclasses have been defined: SimpleEvent, SpatialEvent, and TransitiveEvent. These derived classes are not disjoint, that is, an event can be created by inheriting from multiple classes. These specializations are defined as follows:

- SimpleEvent This is the simplest form of events. A single entity is generally involved and there are no interactions with secondary entities. Relations with other entities are weak and non-mandatory. Examples of this category include the events Starts, Stops, Locks, Unlocks, etc.

- SpatialEvent Describes events that occur in space. Instances of this class have to define at least one of the following properties that have SpatialEvent as domain:

- Location [object reference]: this property allows to specify an instance of the "Background" class, that is the scene, the container that constitutes the environment surrounding the observed entities

- SourceLocation/DestinationLocation [object reference]: this property is set for events that cannot be located in a single place, but occur between two spatially separated locations.

Figure 1 illustrates how the properties of "SpatialEvent" relate with instances of the "Background" class. As can be seen in Figure 2, the "Background" class includes a "GenericPlace" subclass that isn't semantically relevant (e.g. does not correspond to a building, a parking lot, etc.), but can be used to specify absolute topographic data, or an address. The properties of the GenericPlace class are a subset of the Place Description Scheme of MPEG-7. Every instance of GenericPlace can be related through the property "Place" to every instance of objects derived from the "Background" class. Examples of this category include the events Enters/Exits, etc.

- TransitiveEvent This class specifies Events that involve two entities that can be identified as the source of the action and the recipient of the action respectively. The relations between the two entities can be further specified to capture the exact type of interaction. The role of the secondary entity participating in the action has been classified according to a subset of MPEG-7 semantic relations. In this way, recognized events can be 


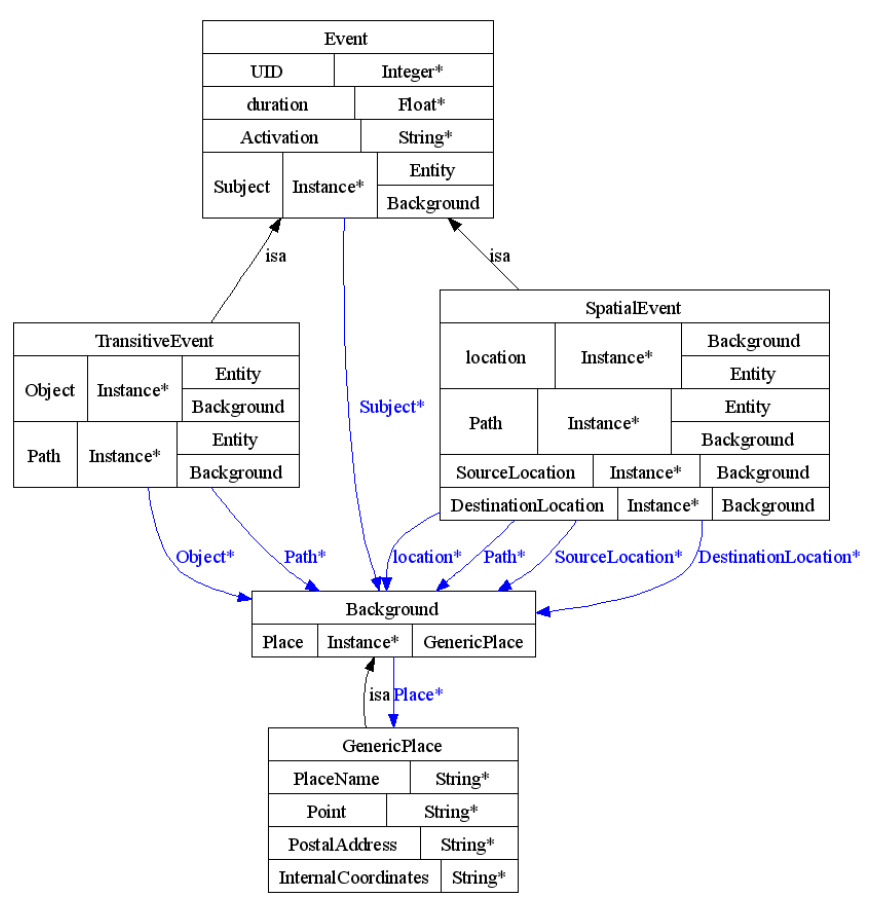

Fig. 1. Some of the relations between the SpatialEvent and Backgroud classes.

straightforwardly annotated by the system. The following properties relate the event to the recipient of the action according to the MPEG-7 definitions:

- Patient [object reference]: the entity suffers the action passively

- Result [object reference]: the entity that results from an event

- Instrument [object reference]: the entity used to carry out the event

- Accompanier [object reference]: the entity participates to the event, without actively starting it

Examples of this category include the events Opens/Closes, Takes/Leaves

\section{VidEO ANNOTATION}

A surveillance system which uses video sensors to collect information about the environment should maintain this information in structured and exchangeable way that can be shared throughout the system for distributed processing. To this end, a Video Event Markup Language (VEML) is also described in [1]. However, in our opinion, it is not clear why this solutions should be adopted in lieu of the MPEG-7 standard which already has these capabilities and is being internationally accepted. In addition, the VEML language is specifically designed for video annotation and it is thus limited to this media. On the contrary, MPEG7, also called "Multimedia Content Description Interface", standardizes the description of generic multimedia content supporting a wide range of applications [11]. Descriptions are comprised of Descriptors (D) and Description Schemes
(DS) that can span from low-level of audio and video (e.g. scale, timbre, instruments, colour, shape, texture) to high-level content meanings (semantics). $\mathrm{C}++$ libraries, such as the one freely available from JOANNEUM Research ${ }^{2}$, can be used to programmatically generate MPEG-7 compliant descriptions.

Here, the purpose is to annotate video streams coming from the sensors and locally processed with descriptions of detected events previously defined in SWRL. In the following example, the "IdentityFraud" event of 1 is detected and recognized in the video-stream produced by a surveillance camera. Only the first, namely "Entity approaching", of the sequence of events leading to an identity fraud, is here reported in Listing 2.

MPEG-7 descriptions can be transmitted in textual or binary format. That is, the standard describes how to encode and decode textual XML descriptions into binary format that could be efficiently compressed and streamed over the network. The "Binary format for MPEG-7" (BiM) was specifically designed to cater for scarce network or storage resources.

An MPEG-7 description can be represented in a tree structure which has a bijective correspondence with the nested syntax of an XML file. Nodes in the tree represent information, while links stand for the "containment" relation. Both "Textual Format for MPEG-7" (TeM) and BiM allow dynamic and incremental transmission of description trees. This means that the standard allows the decomposition of a full description into several fragments (sub-trees), to encapsulate the fragments into Access units, that can be sent over the network separately, and to reconstruct the full description at the destination as shown in Figure 3. This feature is particularly relevant for a system that elaborates video content in real-time, thus building a description as the composition of several temporally separated fragments that have to be sent over the network to other processing units.

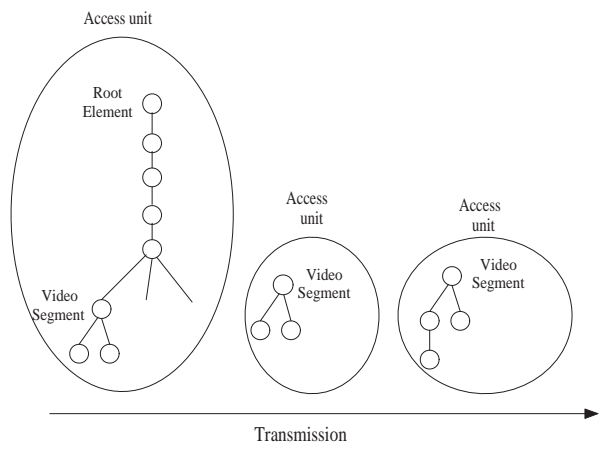

(a)

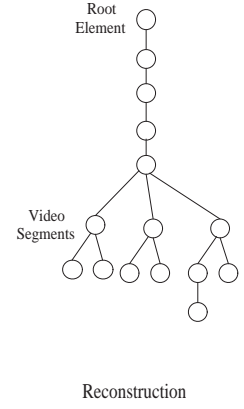

Fig. 3. Description trees and Access units. (a) Fragments of a description tree are sent over the network by first layer nodes. (b) Second layer nodes compose the fragments into a full description.

\section{DISCUSSION}

The approach described in this paper is being experimented as part of an ongoing research on event detection and recognition for video surveillance systems. After investigating proba-

\footnotetext{
${ }^{2}$ http://www.joanneum.at
} 


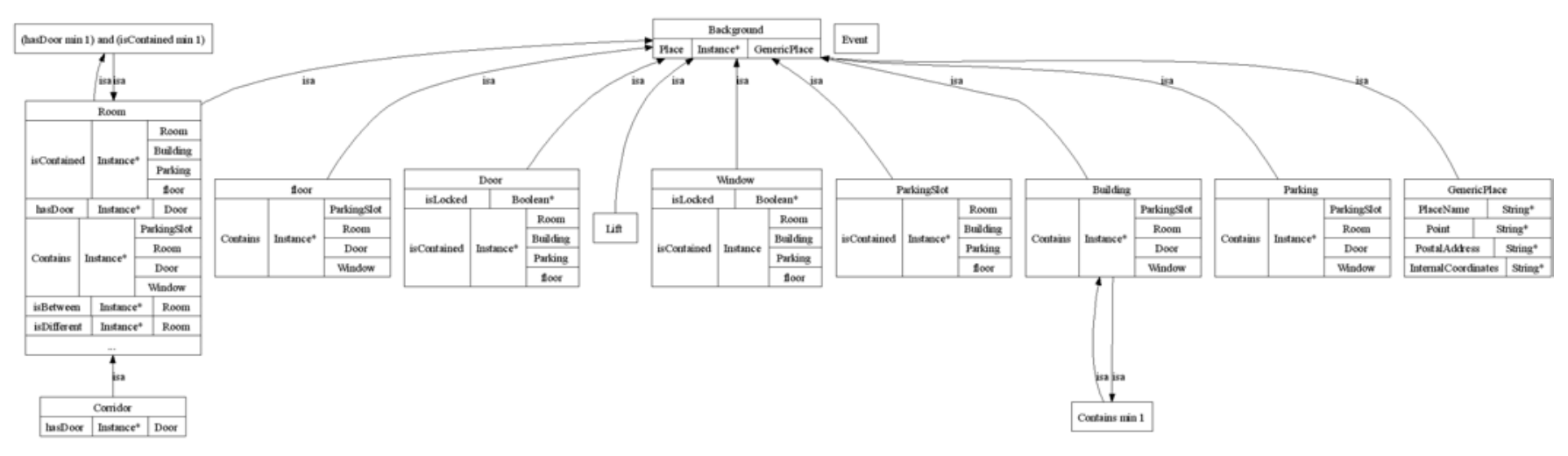

Fig. 2. OntoViz visualization of the Background class used in the experiments. The class comprises structural elements that define the environment of the monitored areas.

bilistic solutions [4], this represents a first attempt to formalize explicitly a priori knowledge for a surveillance system domain. The type of entities and events described here are of course incomplete for a real world scenario, and should serve only to clarify the concepts expressed throughout the paper.

During our experiments, OWL has proven to be a proper language for representing taxonomical contextual information such as entities and background objects. In addition, the availability of a great number of libraries, editors, and reasoners, has considerably simplified the process of defining and continuously checking the consistency of asserted and inferred taxonomical knowledge in our applications.

Regarding inferential knowledge, the definition of complex events as consequents in entailment expressions has proven rather unwieldy. This is in part due to the severely incomplete and buggy implementation of SWRL for OWL that was available. The current implementation, however, is pushing OWL way beyond what was intended for, and it is not clear if the next releases will be able to mimic the power of rule definition languages such as RuleML. A viable solution for building working systems today is still the separation of taxonomical knowledge, expressed in OWL, and inferential knowledge, expressed with specifically designed rule languages such as RuleML or JessML.

\section{Conclusions}

In this paper, we have presented a formalization of domain knowledge relevant to a video surveillance application. In addition to taxonomical knowledge that can can be modelled through OWL ontologies, we have addressed the problem of representing inferential knowledge through the use of the SWRL language. In this way, we have found a way to model complex events as the fusion of simple events. Domain ontologies have been defined according to the classifications given in the MPEG-7 standard in order to straightforwardly annotate video streams according to detected events.

\section{ACKNOWLEDGMENTS}

This work was supported in part by the Italian Ministry of University and Scientific Research within the project PRIN06
(Ambient Intelligence: event analysis, sensor reconfiguration and multimodal interfaces), and in part by the European project SEC6-SA-204400 (HAMLeT).

\section{REFERENCES}

[1] A. R. Francois, R. Nevatia, J. Hobbs, R. C. Bolles, and J. Smith, "VERL: an ontology framework for representing and annotating video events," IEEE MultiMedia Magazine, vol. 12, no. 4, pp. 76-86, Oct.-Dec. 2005.

[2] S. Hongeng, R. Nevatia, and F. Bremond, "Video-based event recognition: activity representation and probabilistic recognition methods," Computer Vision and Image Understanding, vol. 96, no. 2, pp. 129162, November 2004

[3] G. L. Foresti, C. Micheloni, L. Snidaro, P. Remagnino, and T. Ellis, "Active video-based surveillance systems," IEEE Signal Processing Mag., vol. 22, no. 2, pp. 25-37, March 2005.

[4] L. Snidaro, C. Piciarelli, and G. L. Foresti, "Fusion of trajectory clusters for situation assessment," in Proceedings of the Ninth International Conference on Information Fusion, Florence, Italy, July 10-14 2006.

[5] Y. A. Ivanov and A. F. Bobick, "Recognition of visual activities and interactions by stochastic parsing," IEEE Trans. Pattern Anal. Mach. Intell., vol. 22, no. 8, pp. 852-872, August 2000.

[6] T. R. Gruber, "A translation approach to portable ontology specification," Knowledge Acquisition, vol. 5, pp. 199-220, 1993.

[7] C. Matheus, K. Baclawski, M. Kokar, and J. Letkowski, "Using SWRL and OWL to Capture Domain Knowledge for a Situation Awareness Application Applied to a Supply Logistics Scenario," in Proceedings of International Conference on Rules and Rule Markup Languages for the Semantic Web, Galway, Ireland, November 2005.

[8] N. M. Oliver, B. Rosario, and A. P. Pentland, "A Bayesian computer vision system for modeling human interactions," IEEE Transactions on Pattern Analysis and Machine Intelligence, vol. 22, no. 8, pp. 831-843, 2000.

[9] M. J. O'Connor, H. Knublauch, S. W. Tu, B. Grossof, M. Dean, W. E. Grosso, and M. A. Musen, "Supporting rule system interoperability on the semantic web with swrl," in Proceedings of the Fourth International Semantic Web Conference (ISWC2005), Galway, Ireland, 2005.

[10] R. Orchard, "Fuzzy Reasoning in JESS: The Fuzzyj Toolkit and Fuzzyjess," in Proceedings of the Third International Conference on Enterprise Information Systems (ICEIS), Setubal, Portugal, July 7-10 2001, pp. $533-542$.

[11] B. Manjunath, P. Salembier, and T. Sikora, Eds., Introduction to MPEG7: Multimedia Content Description Interface. New York, NY, USA: John Wiley \& Sons, Inc., 2002. 
Listing 2

EXAMPLE OF MPEG-7 ANNOTATION

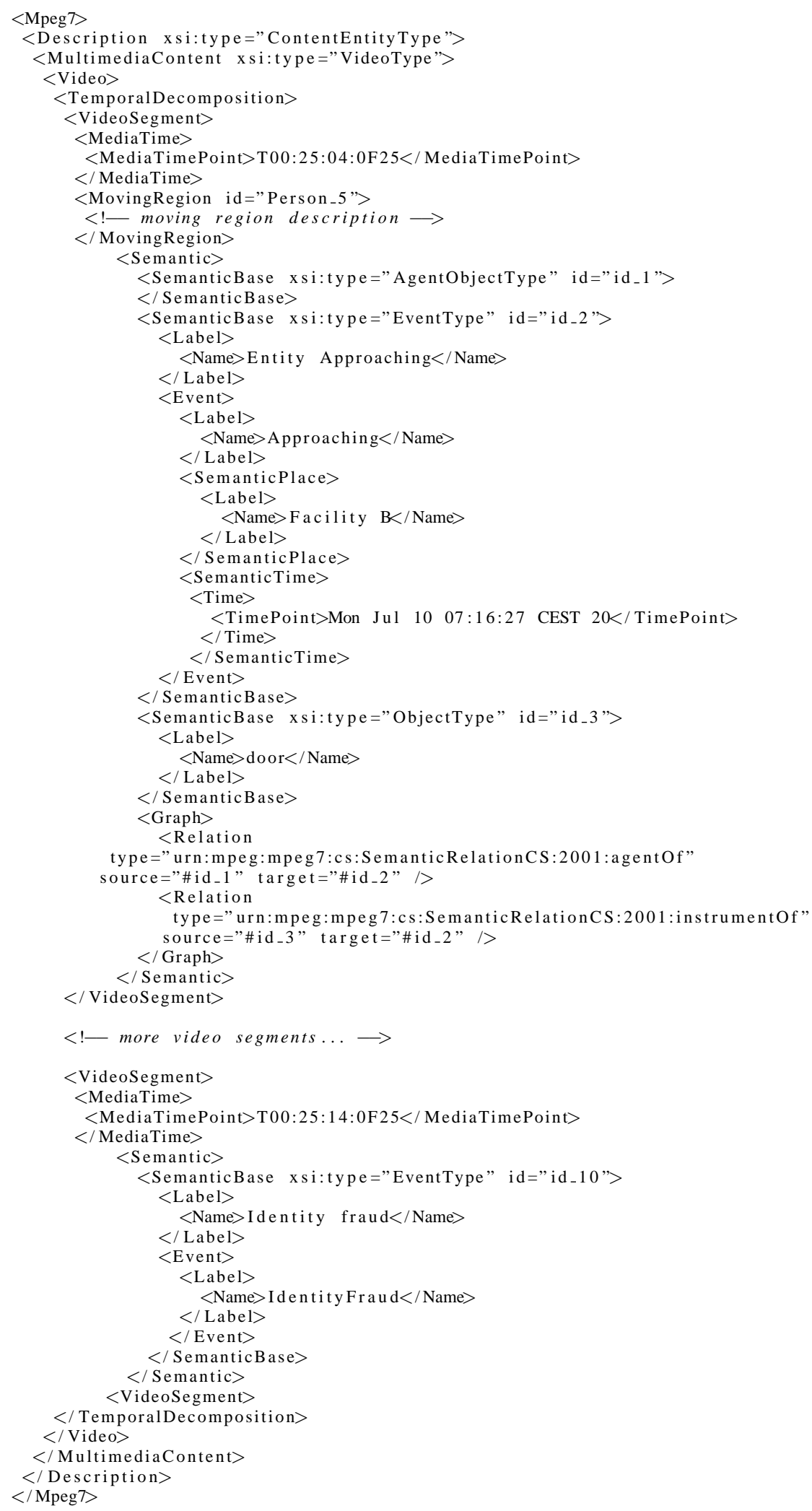

